\title{
Neural lineage-specific homeoprotein BRN2 is directly involved in TTF1 expression in small-cell lung cancer
}

\author{
Masashi Sakaeda ${ }^{1,2}$, Hanako Sato ${ }^{3}$, Jun Ishii ${ }^{1,2}$, Chie Miyata ${ }^{3}$, Hiroshi Kamma ${ }^{2}$, Yukiko Shishido-Hara ${ }^{2}$, \\ Hiroaki Shimoyamada ${ }^{2}$, Masachika Fujiwara ${ }^{2}$, Tetsuya Endo ${ }^{1,4}$, Ryota Tanaka ${ }^{5}$, Haruhiko Kondo ${ }^{5}$, Tomoyuki Goya ${ }^{5}$, \\ Ichiro Aoki ${ }^{1}$ and Takuya Yazawa ${ }^{2}$
}

Thyroid transcription factor 1 (TTF1) plays crucial roles in thyroid, lung, and developing brain morphogenesis. Because TTF1-expressing neoplasms are generated from organs and tissues that normally express TTF1, such as the thyroid follicular epithelium and peripheral lung airway epithelium, TTF1 is widely used as a cell lineage-specific and diagnostic marker for thyroid carcinomas and for lung adenocarcinomas with terminal respiratory unit (TRU) differentiation.

However, among lung neuroendocrine tumors, small-cell carcinomas (small-cell lung cancers (SCLCs)), most of which are generated from the central airway, also frequently express TTF1 at high levels. To clarify how SCLCs express TTF1, we investigated the molecular mechanisms of its expression using cultivated lung cancer cells and focusing upon neural cellspecific transcription factors. Both SCLC cells and lung adenocarcinoma cells predominantly expressed isoform 2 of TTF1, and TTF1 promoter assays in SCLC cells revealed that the crucial region for activation of the promoter, which is adjacent to the transcription start site of TTF1 isoform 2, has potent FOX-, LHX-, and BRN2-binding sites. Transfection experiments using expression vectors for FOXA1, FOXA2, LHX2, LHX6, and BRN2 showed that BRN2 substantially upregulated TTF1 expression, whereas FOXA1/2 weakly upregulated TTF1 expression. BRN2 and FOXA1/2 binding to the TTF1 promoter was confirmed through chromatin immunoprecipitation experiments, and TFF1 expression in SCLC cells was considerably downregulated after BRN2 knockdown. Furthermore, the TTF1 promoter in SCLC cells was scarcely methylated, and immunohistochemical examinations using a series of primary lung tumors indicated that TTF1 and BRN2 were coexpressed only in SCLC cells. These findings suggest that TTF1 expression in SCLC is a cell lineage-specific phenomenon that involves the developing neural cell-specific homeoprotein BRN2.

Laboratory Investigation (2013) 93, 408-421; doi:10.1038/labinvest.2013.2; published online 28 January 2013

KEYWORDS: BRN2; homeoprotein; small-cell lung cancer; TTF1

Thyroid transcription factor 1 (TTF1), also known as thyroid-specific enhancer-binding protein (T/EBP) or NKX2.1, is a homeodomain-containing transcription factor that plays crucial roles in thyroid, lung, and developing brain morphogenesis. ${ }^{1-3}$ TTF1 is responsible for the transcriptional activation of thyroglobulin, thyroperoxidase, and thyrotropin receptor in the thyroid and of surfactant and Clara cell secretory proteins in the lung. ${ }^{4-6}$ In the normal adult lung, TTF1 is stably expressed in peripheral lung epithelial cells such as nonciliated bronchiolar epithelial cells (Clara cells) and type II pneumocytes and bronchiolar basal cells. ${ }^{7}$

TTF1 expression in tumors is exclusively found in lung and thyroid tissues. ${ }^{8,9}$ Among lung cancers, $>70 \%$ of adenocarcinomas express TTF1, whereas TTF1 expression is found at a very low frequency in squamous cell carcinomas and large-cell carcinomas. ${ }^{10-12}$ Most lung adenocarcinomas arise in the peripheral airway, and it has been reported that lung adenocarcinomas with terminal respiratory unit (TRU) differentiation (nonciliated bronchiolar epithelial cell-like and/or type II pneumocyte-like cellular morphology) reveal frequent TTF1 positivity. ${ }^{12,13}$ Therefore, TTF1 is used as a lineage marker for TRU in lung carcinogenesis and as a diagnostic marker for lung adenocarcinomas with TRU differentiation.

Small-cell carcinoma of lung (small-cell lung cancer (SCLC)) is one of the most aggressive neoplasms in humans

\footnotetext{
${ }^{1}$ Department of Pathology, Yokohama City University Graduate School of Medicine, Yokohama, Kanagawa, Japan; ${ }^{2}$ Department of Pathology, Kyorin University School of Medicine, Mitaka, Tokyo, Japan; ${ }^{3}$ Department of Anatomy, St Marianna University School of Medicine, Kawasaki, Kanagawa, Japan; ${ }^{4}$ Department of General Thoracic Surgery, Jichi Medical University, Shimotsuke, Tochigi, Japan and ${ }^{5}$ Department of Surgery, Kyorin University School of Medicine, Mitaka, Tokyo, Japan Correspondence: Dr T Yazawa, Department of Pathology, Kyorin University School of Medicine, 6-20-2 Shinkawa, Mitaka, Tokyo 181-8611, Japan. E-mail: tkyazawa@ks.kyorin-u.ac.jp 
and is categorized as a neuroendocrine tumor. ${ }^{14}$ SCLC is often generated from the central airway epithelium, which does not express TTF1, in contrast to most lung adenocarcinomas. ${ }^{14}$ However, paradoxically, $>90 \%$ of SCLCs express high levels of TTF $1 .{ }^{15}$ To date, the expression mechanism of TTF1 in SCLC has been unresolved.

The diverse cell types present in adult organisms are produced during development by the action of cell lineagespecific transcription factors that define cell type-specific gene expression patterns. Transcription factors activate or repress gene expression by recognizing the sequence of DNA base sequences in gene promoter regions. It has been reported that TTF1 promoter activation in human peripheral lung epithelial cells and mouse lung adenocarcinoma cells is maintained by the combinational or cooperative actions of transcription factors such as forkhead box A1 (FOXA1, also named hepatocyte nuclear factor $3 \alpha$ (HNF3 $\alpha)$ ), FOXA2 $\left(\right.$ HNF3 $\beta$ ), and GATA6. ${ }^{16,17}$ Although the SCLC cell of origin has not been formally identified, there are some reports supporting the hypothesis that cells of the neuroendocrine cell lineage give rise to SCLC. ${ }^{18,19}$ These reports imply the existence of a neuroendocrine cell lineage-specific expression mechanism of TTF1 in SCLC.

SCLC overexpresses a proneural cell-specific transcription factor, achaete-scute homolog like 1 (ASCL1), suggesting that SCLC possesses developmental neural cell phenotypes as well as neuroendocrine phenotypes. ${ }^{20}$ Because TTF1 is an important transcription factor for fetal diencephalon morphogesesis, ${ }^{1}$ there is a possibility that TTF1 expression in SCLC is regulated by developmental neural cell-specific transcription factor(s). The POU domain family of transcription factors is essential for organ development, and class III and IV POU domain transcription factors are strictly expressed in the developing nervous system. BRN2, a member of the class III POU domain transcription factors, has been reported to play crucial roles in the development of the endocrine hypothalamus, which is a part of diencephalon. ${ }^{21,22}$ A previous report that BRN2 is expressed in SCLC indicates a possible role of BRN2 in TTF1 expression regulation in SCLC. ${ }^{23}$

Cellular phenotypes are stabilized by epigenetic modifications that allow for the faithful transmission of cell typespecific gene expression patterns; DNA hypermethylation often occurs in cancer cells and is important for gene silencing in carcinogenesis. ${ }^{24}$ Because TTF1 plays a crucial role in organ development and because the TTF1 gene promoter possesses CpG islands, the TTF1 gene may be epigenetically modified. It was recently reported that TTF1 expression deficiency in thyroid cancers is caused by epigenetic modification in association with cancer progression..$^{25}$ However, there have been no studies on TTF1 gene methylation in lung cancers.

To elucidate the mechanisms by which TTF1 is expressed in SCLC, we searched for key transcriptional factors that regulate TTF1 expression and investigated possible epigenetic modifications of the TTF1 gene. Here, we present data showing that BRN2, an important transcription factor in neural cell differentiation, is expressed and activates the TTF1 gene and that the TTF1 promoter is not epigenetically modulated in SCLC cells.

\section{MATERIALS AND METHODS Cultured Lung Cancer Cells}

In all, 16 human lung cancer cell lines were used in this study: 8 small-cell carcinoma cell lines, TKB12, TKB15, TKB16, TKB17, Lu134A, Lu135, Lu139, and H1688; 4 adenocarcinoma cell lines, LC2/ad, H441, TKB14, and A549; 2 squamous cell carcinoma cell lines, TKB4 and TKB7; and 2 large-cell carcinoma cell lines, TKB1 and TKB5. ${ }^{26-31}$ H1688, H441, and A549 were purchased from the American Type Culture Collection (ATCC, Manassas, VA, USA), and LC2/ad was obtained from the Riken Bioresource Center (Ibaraki, Japan). Non-small-cell lung cancer (NSCLC) cells were cultivated in Dulbecco's modified Eagle's medium, and SCLC cells were cultured in RPMI-1640 medium. The media were supplemented with $10 \%$ heat-inactivated fetal calf serum, $100 \mathrm{U} / \mathrm{ml}$ penicillin, and $100 \mu \mathrm{g} / \mathrm{ml}$ streptomycin. The cells were maintained at $37^{\circ} \mathrm{C}$ in $5 \% \mathrm{CO}_{2}$.

\section{Western Blot Analysis}

Whole-cell lysates (50 $\mu \mathrm{g}$ protein/lane) were separated on $12.5 \%$ SDS-PAGE gels and transferred to nitrocellulose membranes (Schleicher \& Schell, Keene, NH, USA). The membranes were blocked for $1 \mathrm{~h}$ at room temperature with $1 \%$ skim milk in PBS containing $0.1 \%(\mathrm{v} / \mathrm{v})$ Tween 20 (PBS-T) and then incubated with diluted mouse monoclonal anti-TTF1 (M3575 clone 8G7G3/1; Dako, Copenhagen, Denmark), goat polyclonal anti-FOXA1 (sc-9186; Santa Cruz Biotechnology, Santa Cruz, CA, USA), goat polyclonal antiFOXA2 (sc-6554; Santa Cruz Biotechnology), goat polyclonal anti-LHX2 (sc-19344; Santa Cruz Biotechnology), rabbit polyclonal anti-LHX6 (sc-98607; Santa Cruz Biotechnology), or goat polyclonal anti-BRN2 antibody (ab101726; Abcam, Cambridge, UK) for $1 \mathrm{~h}$ at room temperature. The dilution ratios of the primary antibodies were 1:1000 for TTF1, 1:2000 for FOXA1, FOXA2, LHX2, and LHX6, and 1:5000 for BRN2. After three 10-min washes with PBS-T at room temperature, the membranes were incubated at room temperature for 30 min with a diluted peroxidase-labeled secondary antibody against mouse (NA931V; GE Healthcare, Buckinghamshire, UK), goat (sc-2741; Santa Cruz Biotechnology), or rabbit (NA933V; GE Healthcare). The membranes were then washed three times for $10 \mathrm{~min}$ with PBS-T at room temperature, and immunopositive signals were visualized using an enhanced chemiluminescence detection kit (ECL; GE Healthcare). Mouse monoclonal anti- $\beta$-actin (A5441; SigmaAldrich, St Louis, MO, USA) was used as an internal control. The anti- $\beta$-actin antibody was diluted 1:10 000 . 


\section{TTF1 Promoter Assay}

Luciferase vectors (pGL4.10luc2; Promega, Madison, WI, USA) containing TTF1 promoter fragments of various lengths were constructed. The promoter fragments were generated using PCR amplification with specific primer sets (Table 1), genomic DNA extracted from TKB15 cells, and a TaKaRa LATaq DNA polymerase (Takara, Shiga, Japan). The PCR products were cloned into pT7Blue (Novagen, Madison WI, USA), sequenced, and inserted into pGL4.10luc2. For mutational analyses, a segment containing the full-length promoter sequence of TTF1 was removed using KpnI and Aor $51 \mathrm{HI}$ and replaced with synthetic double-stranded oligonucleotide inserts containing mutated sequences. Changes from the wild-type to mutant sequences (sense strand) were as follows: TGTTT to GTAGA for the potent FOX-binding site and TAATT to TCCTT for the potent LHX/ BRN2-binding site.

The cultured lung cancer cells were transiently cotransfected with the promoter sequence-inserted luciferase vectors and the control vector (pGL4.74TK; Promega) using FuGENE6 transfection reagent (Roche Molecular Biochemicals, Indianapolis, IN, USA) according to the manufacturer's instructions. The cells were seeded at $50 \%$ confluency in sixwell plates, cultivated for $24 \mathrm{~h}$, and then cotransfected with $1 \mu \mathrm{g} /$ well reporter plasmid vector and $50 \mathrm{ng} /$ well pGL4.74TK. After $24 \mathrm{~h}$, the cells were washed three times with PBS, and cell lysates were prepared and subjected to luciferase assays. Luciferase activity was measured using a luminometer (Turner Biosystems, Sunnyvale, CA, USA) and the DualLuciferase Reporter Assay System (Promega) according to the manufacturer's instructions. The data reflect the mean + s.d. from three independent experiments performed in triplicate.

\section{Reverse Transcriptase-PCR (RT-PCR) and Quantitative RT-PCR (qRT-PCR)}

Total RNA was extracted from the cells using TRIzol (Invitrogen, Carlsbad, CA, USA). Complementary DNA (cDNA) synthesis, and subsequent PCR reactions were performed using $500 \mathrm{ng}$ of DNase-treated total RNA and an RNA-LAPCR kit (Takara) according to the manufacturer's instructions. $\beta$-Actin served as an internal control. The number of PCR cycles was set at 35 for TTF1, FOXA1, FOXA2, BRN2, LHX1, LHX2, LHX3, LHX4, LHX5, LHX6, LHX8, LHX9, and GATA6 and at 22 for $\beta$-actin. The nucleotide sequences of the forward and reverse primers are listed in Table 1. The suitability of these primer sets for PCR was assured by Takara.

For qRT-PCR, cDNA was synthesized from DNase-treated total RNA using the SuperScript First-Strand Synthesis System according to the manufacturer's instructions (Invitrogen). The cDNA generated was used as a template in real-time PCR reactions with Fast SYBR Green Master Mix (no. 4385612, Applied Biosystems, Carlsbad, CA, USA). The reactions were run on a StepOnePlus real-time PCR system (Applied Biosystems). The primer sets used for TTF1 and $\beta$-actin are listed in Table 1 . The means and s.d. of the relative copy numbers relative to $\beta$-actin mRNA were obtained from triplicate PCR reactions.

\section{Construction of Retroviral Vectors and Transfection}

We used a retrovirus-based Tet-On Advanced Inducible Gene Expression System (pTet-On Advanced and pRevTRE; Clontech, Mountain View, CA, USA) to avoid difficulty in obtaining stable transfectants because of unexpected growth inhibitory effects caused by the transfected genes. ${ }^{27} \mathrm{We}$ selected TKB5 and TKB1 cells for transfection because these cells express undetectable or low levels of FOXA1, FOXA2, BRN2, LHX2, and LHX6. In addition, we selected A549 cells for transfection because A549 cells express undetectable levels of TTF1 in spite of an unmethylated TTF1 promoter (see Results and Supplementary Figure 1). A pTet-On Advanced vector (Clontech) that expresses reverse tetracycline-controlled transactivator protein (rtTA) was initially transfected into TKB5, TKB1, or A549 cells using the FuGENE6 transfection reagent (Roche Molecular Biochemicals). After neomycin $(1 \mathrm{mg} / \mathrm{ml})$-based selection for 3 weeks, stable transfectants were cloned. Next, the TTF1, FOXA1, FOXA2, LHX2, LHX6, and BRN2 cDNA-containing pRevTRE vectors were constructed using the respective cDNAinserted cloning vector (pMD20; Takara). The respective cDNAs were synthesized from DNase-treated total RNA using the SuperScript First-Strand Synthesis System according to the manufacturer's instructions (Invitrogen) and amplified by PCR using a specific primer set (Table 1) and primeSTAR HS DNA polymerase (Takara). After sequencing, the desired cDNA-inserted pRevTRE vector ( $\mathrm{pTTF} 1$ isoform 1, pTTF1 isoform 2, pFOXA1, pFOXA2, pLHX2, pLHX6, or pBRN2) or an empty pRevTRE vector (pEMPTY) was transfected into HEK-GP-293 cells using FuGENE6 (Roche Molecular Biochemicals). After $24 \mathrm{~h}$, the conditioned medium was recovered as a viral solution. The desired genes were introduced by incubating pTet-On Advanced-transfected TKB5 cells with the viral solution and $10 \mu \mathrm{g} / \mathrm{ml}$ polybrene (Sigma-Aldrich) for $24 \mathrm{~h}$. The cells were selected with hygromycin $(500 \mu \mathrm{g} / \mathrm{ml})$ for 3 weeks. To induce expression of the desired genes, the transfectants were cultivated in medium with $1 \mu \mathrm{g} / \mathrm{ml}$ doxycycline (Clontech) for $48 \mathrm{~h}$ before harvest. To validate the association of BRN2 with TTF1 expression, we established BRN2-inducible TKB1 and A549 cells using the same methodology. Recombinant TTF1 isoform 1 and 2 proteins used as positive controls in the western blot analysis were prepared through the transient transfection of pTTF1 isoform 1 and pTTF1 isoform 2 into stably pTet-On Advanced-transfected HEK-GP-293 cells.

To conduct a retrovirus-based siRNA experiment targeted to BRN2, two types of synthetic double-stranded oligonucleotide inserts specific for the production of BRN2 siRNAs were ligated in tandem with a promoter cassette and a linearized pSINsiDKI vector (Takara). The sequences of the $B R N 2$ siRNA inserts and nonspecific scrambled control 
Table 1 Primer sets used in this study

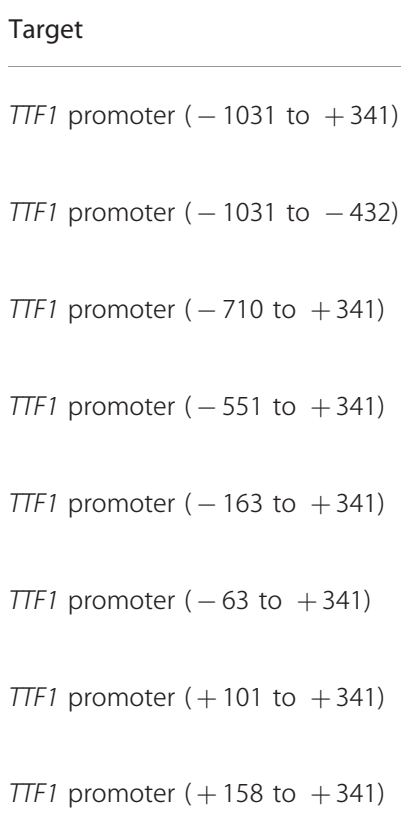

TTF1 (for RT-PCR and qRT-PCR)

FOXA1 (for RT-PCR)

FOXA2 (for RT-PCR)

LHX1 (for RT-PCR)

LHX2 (for RT-PCR)

LHX3 (for RT-PCR)

LHX4 (for RT-PCR)

LHX5 (for RT-PCR)

LHX6 (for RT-PCR)

LHX8 (for RT-PCR)

LHX9 (for RT-PCR)

PAX4 (for RT-PCR)

HOXB3 (for RT-PCR)

GATA6 (for RT-PCR)
F 5'-CAACAGACAGACGGGCACTCA-3'

R 5'-GATTCGGCGGCGGCTGGAGGA-3'

F 5'-CAACAGACAGACGGGCACTCA-3'

600

R 5'-CGGGCTTCGCTGCGCTGAGCC-3'

F 5'-GCCCACTGAAAAACCCCTGAG-3'

1051

R 5'-GATTCGGCGGCGGCTGGAGGA-3'

F 5'-GGCGGCAGAAGAGAGGCAGAC-3'

896

R $5^{\prime}$-GATTCGGCGGCGGCTGGAGGA-3'

F 5'-CTCTCGGTTCCCTCCTCCTTC-3'

504

R 5'-GATTCGGCGGCGGCTGGAGGA-3'

F 5'-ATGCTITGGGTCTCGTCTCTG-3'

404

R $5^{\prime}$-GATTCGGCGGCGGCTGGAGGA-3'

F 5'-TCGGGGCTCCGCTGGCGGCGT-3'

R 5'-GATTCGGCGGCGGCTGGAGGA-3'

F 5'-TGCAGCAGCACGCCGTGGGGC-3'

184

R 5'-GATTCGGCGGCGGCTGGAGGA-3'

F 5'-GCTACTGCAACGGCAACCTG-3'

168

R 5'-CCATGCCGCTCATGTTCA-3'

F 5'-GCTACTACGCAGACACGCAGGA - $3^{\prime}$

101

R 5'-CATGGTCATGTAGGTGTTCATGGAG-3'

F 5'-CGTCCGACTGGAGCAGCTACTAT-3'

66

R 5'-CGGCGTTCATGTTGCTCAC-3'

F 5'-TGCGTCCAGTGCTGTGAATG-3'

186

R 5'-GAAGCAGTTCAGGTGAAACACTाTG-3'

F 5'-ACCTGTTCAGCAAGGACGGTAG-3'

152

R 5'-GTGAAGCAGTTGAGGTGATAAACCA-3'

F 5'-AGACATGAAATGTCACCTTGCTGAG-3'

R 5'-TCGACAGGCAAGGCCAATC-3'

F 5'-TGCACTGCTTTGCTTGCATC-3'

142

R 5'-CGCTTAGCTCCAGCCTCTGA-3'

F 5'-CGTGTGCAAAGACGACTACCTGA-3'

R 5'-AACTGCGGTCCGTACAGGATG-3'

F 5'-CTCTGCCGCATCCACTACGA-3'

190

R 5'-TGTTGTCCTGCGCGAACTG-3'

F 5'-ATGCAAGCACAATITGCTCAGG-3'

R 5'-TGGCGTGCTCTACAATTCTGAAAC-3'

F 5'-GGGAGTGGACATCGTCAATTACAAC-3'

R 5'-CGGAGCTGGTGATGCTTGAA-3'

F 5'-CAAATGGCGTCGGCAAGA-3'

R 5'-GGGCAACCCTTGGTACAGTCA-3'

F 5'-CCTTCGTCATGAATGGGATCTG-3'

R 5'-GTCTGTTCCAAGCGGCTGA-3'

F 5'-GCGGGCTCTACAGCAAGATGA-3'

107 
Table (Continued)

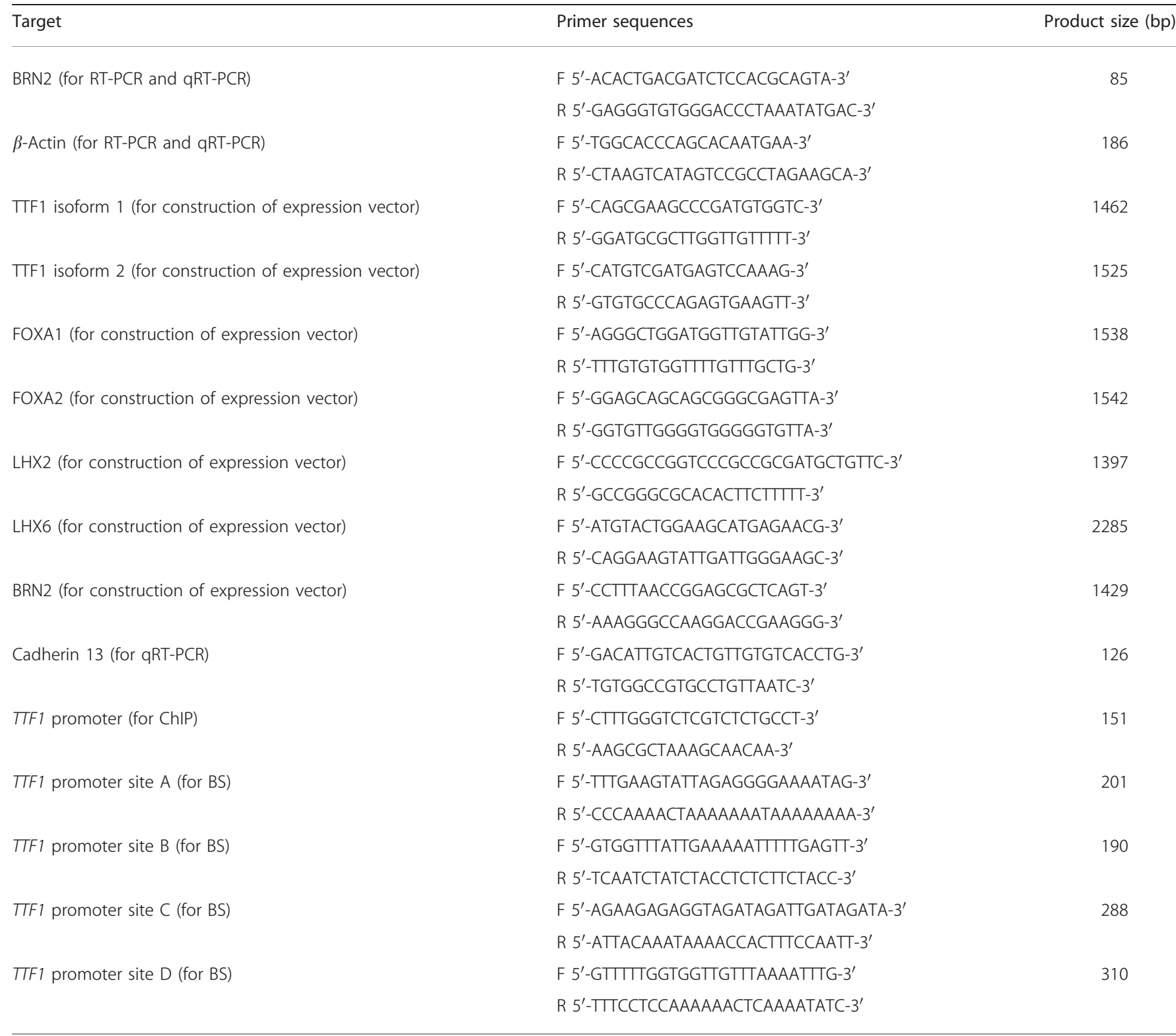

Abbreviations: BS, bisulfite sequencing; ChIP, chromatin immunoprecipitation; qRT-PCR, quantitative RT-PCR; F, forward primer sequence; R, reverse primer sequence.

(NSC) siRNA inserts are shown in Table 2. The siRNAproducing vectors, psiBRN2 and psiNSC, were transfected into HEK-GP2-293 cells with FuGENE6 (Roche Molecular Biochemicals); the desired genes were then introduced by incubating TKB15 or Lu139 cells (representatives of SCLC cell lines) with the viral solution containing $10 \mu \mathrm{g} / \mathrm{ml}$ polybrene (Sigma-Aldrich) for $24 \mathrm{~h}$. The cells were selected with neomycin $(1 \mathrm{mg} / \mathrm{ml})$ for 3 weeks before being used for experiments.

Because it has been reported that BRN2 is a transcriptional repressor of cadherin 13 (CDH13), ${ }^{32}$ we investigated changes in $\mathrm{CDH} 13$ mRNA expression levels after the forced expression/repression of BRN2 in lung cancer cells to examine whether pBRN2 and psiBRN2 functioned correctly. The CDH13-specific primer set is listed in Table 1.

\section{Chromatin Immunoprecipitation (ChIP) Assay}

A ChIP assay was performed using TKB15 cells (a representative SCLC cell line) and a kit (no. 9003; Cell Signaling Technology, Beverly, MA, USA) according to the manufacturer's instructions. Briefly, TKB15 cells were crosslinked by adding formaldehyde to the culture medium at a final concentration of $1 \%(\mathrm{~V} / \mathrm{V})$. After a 10 -min fixation, the cells were washed with cold PBS, collected, and lysed with lysis buffer. The nuclei were collected and treated with micrococcal nuclease to digest the DNA. After stopping 
Table 2 Oligonucleotide sequences for the construction of psi-BRN2 and psi-NSC vectors

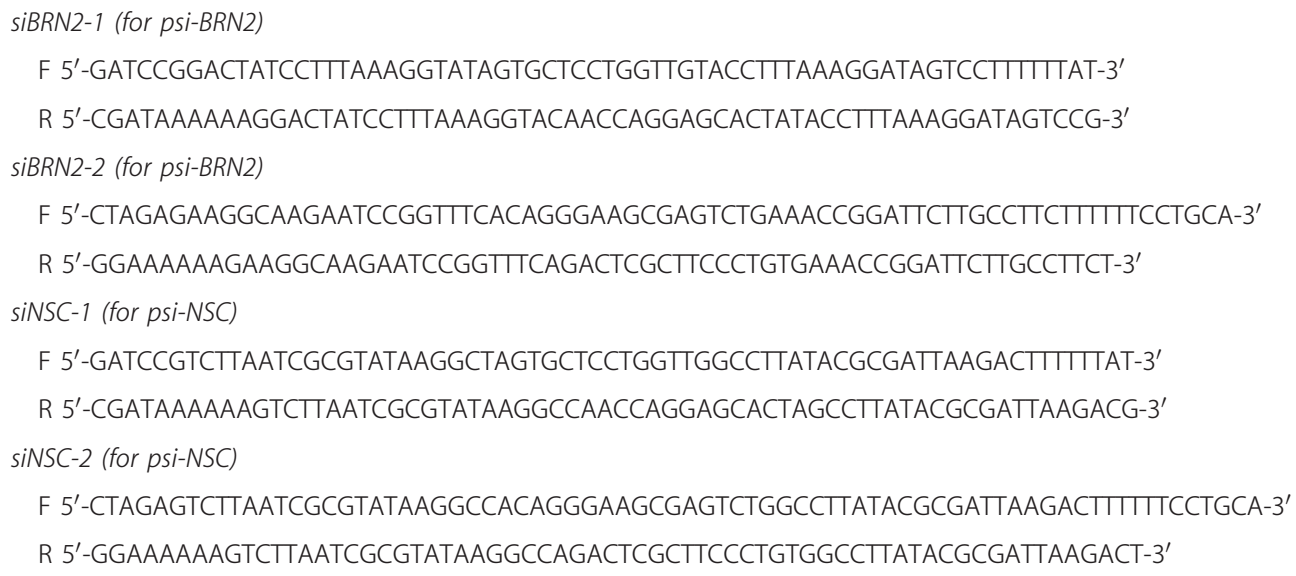

Abbreviations: $F$, forward sequence; NSC, nonspecific scrambled control; R, reverse sequence.

digestion by adding EDTA, sonicating, and centrifuging, soluble material was obtained as the chromatin solution. The chromatin solution was incubated with $5 \mu \mathrm{g}$ of the following antibodies: goat anti-FOXA1 (sc-9186; Santa Cruz Biotechnology), goat anti-FOXA2 (sc-6554; Santa Cruz Biotechnology), goat anti-LHX2 (sc-19344; Santa Cruz Biotechnology), rabbit anti-LHX6 (sc-98607; Santa Cruz Biotechnology), goat anti-BRN2 (sc-6029; Santa Cruz Biotechnology), normal goat IgG (sc-2028; Santa Cruz Biotechnology), or normal rabbit IgG (sc-2027; Santa Cruz Biotechnology). The antibody/chromatin mixtures were precipitated with protein $G$ magnetic beads, and the beads were sequentially washed with ChIP wash buffer for each ChIP reaction. The chromatin was treated with ChIP elution buffer and eluted from the antibody-protein $G$ magnetic beads. Crosslinking was reversed through the addition of $\mathrm{NaCl}$ and proteinase $\mathrm{K}$, and DNA was purified using spin columns. The forward and reverse PCR primers used to detect target sequences containing the potent FOX-, LHX-, and BRN2-binding sites are described in Table 1.

\section{Bisulfite Sequencing}

Genomic DNA extracted from lung cancer cell lines was treated with sodium bisulfite using a MethylEasy DNA bisulfite modification kit (Human Genetic Signatures, Macquarie Park, Australia), according to the manufacturer's instructions. Genomic DNA $(1 \mu \mathrm{g} / 20 \mu \mathrm{l})$ was denatured with $\mathrm{NaOH}$ (final concentration, $0.273 \mathrm{M}$ ) for $15 \mathrm{~min}$ at $37^{\circ} \mathrm{C}$ and then treated with $220 \mu$ l of combined reagent 1 and reagent 2 for $16 \mathrm{~h}$ at $55^{\circ} \mathrm{C}$. The bisulfite-modified DNA was purified, precipitated, resuspended in reagent 3 , and used for bisulfite sequencing. Four primer sets were constructed using promoter sequence data and primer design software (Table 1).

\section{Treatment with 5-Aza-2'-deoxycytidine and Trichostatin A}

The cells were treated with either $10 \mu \mathrm{M}$ 5-aza-2'deoxycytidine (AZA; Wako, Tokyo, Japan), which is a DNA methyltransferase inhibitor, for $72 \mathrm{~h}$ by exchanging the medium everyday or with $300 \mathrm{ng} / \mathrm{ml}$ trichostatin A (TSA; Wako), a histone deacethylase inhibitor, for $24 \mathrm{~h}$. After treatment, total RNA was extracted, and quantitative RT-PCR was conducted as reported previously. ${ }^{26}$ The equivalent volume of vehicle, 50\% acetic acid for AZA (VCL1) or 100\% ethanol for TSA (VCL2), was applied as a control.

\section{Immunohistochemistry}

The institutional ethical board of Kyorin University School of Medicine approved the examination of a series of 17 surgically resected primary lung tumor tissues. Immunohistochemistry was conducted using formalin-fixed, paraffin-embedded tissue sections of five SCLCs, five adenocarcinomas, five squamous cell carcinomas, and two typical carcinoid tumors. Consecutive tissue sections were stained with hematoxylin and eosin (HE) or immunostained using mouse monoclonal anti-TTF1 (M3575, clone 8G7G3/1; Dako), goat polyclonal anti-BRN2 (ab101726; Abcam), goat polyclonal anti-FOXA1 (sc-9186; Santa Cruz Biotechnology), goat polyclonal anti-FOXA2 (sc-6554; Santa Cruz Biotechnology), goat polyclonal anti-LHX2 (sc-19344; Santa Cruz Biotechnology), or rabbit polyclonal anti-LHX6 (sc-98607; Santa Cruz Biotechnology) antibody and an Envision detection system (Dako) after autoclave treatment to retrieve antigenicity of tissue sections. The dilution rates of the primary antibodies were 1:100 for TTF1, 1:200 for FOXA1, FOXA2, LHX2, and LHX6, and 1:500 for BRN2. Negative control immunostaining was performed with nonimmunized mouse, goat, or rabbit IgG (Santa Cruz Biotechnology) in place of the primary antibody. 
Immunostaining was detected with diaminobenzidine (Dako), and the nuclei were counterstained with hematoxylin. The immunoreactivity was evaluated using a scoring system from 0 to 2: score 0 indicates no staining; score 1 indicates $1-50 \%$ positive cells; score 2 indicates $>50 \%$ positive cells.

\section{RESULTS}

\section{TTF1 Expression in Cultivated Lung Cancer Cells}

TTF1 expression was found in all SCLC cell lines and two of four adenocarcinoma cell lines examined (Figure 1). SCLC and adenocarcinoma cells mainly expressed isoform 2 of TTF1. The amount of TTF1 in the SCLC cell lines was greater than that in the adenocarcinoma cell lines. We also obtained consistent qRT-PCR results for TTF1 protein expression levels in the two types of cell lines (data not shown). However, two of the four adenocarcinoma cell lines, the squamous cell carcinoma cell lines, and the large-cell carcinoma cell lines did not express detectable levels of TTF1.

\section{Crucial Region for TTF1 Promoter Activation in SCLC}

To identify the region of the TTF1 promoter required for gene activation, we conducted luciferase assays using full-length and truncated TTF1 promoter sequence-inserted luciferase vectors. Because the TTF1 expressed in cultivated lung cancer cells was mainly isoform 2, $1372 \mathrm{bp}$ of the region upstream from the translation start site of isoform 2 was defined as the TTF1 gene promoter using computer software (Gene2Promoter, Genomatix, Munich, Germany; Figure 2a). When tested with vectors containing the full-length or partial promoter sequences, TKB15 and Lu139 cells, which were used as SCLC representatives, revealed very similar luciferase activity patterns (Figure $2 \mathrm{~b}$ ). The TTF1 isoform 1-regulating promoter sequence-inserted luciferase vector $(-1031$ to -432; the number indicates the nucleotide position from the transcription start site $(+1)$ of the TTF1 isoform 2 gene) had $\sim 18-22 \%$ of the promoter activity of the full-length promoter sequence-inserted luciferase vector. The luciferase activity did not significantly change even when up to $967 \mathrm{bp}$ of the $5^{\prime}$-terminal sequence of the TTF1 gene promoter was

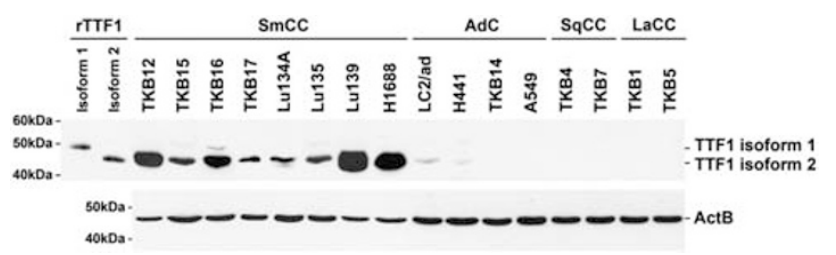

Figure 1 Western blot analysis of TTF1 proteins. Small-cell carcinoma (SmCC) cell lines expressed abundant TTF1. Weak TTF1 signals were found in two of four adenocarcinoma (AdC) cell lines, whereas two AdC cell lines and all squamous cell carcinoma (SqCC) and large-cell carcinoma (LaCC) cell lines examined were TTF1 negative. TTF1 isoform 2 was predominantly expressed in lung cancer cells. Recombinant TTF1 proteins ( $r T T F 1$ isoform 1 and isoform 2) were used as positive controls; $\beta$-actin (ActB) served as an internal control. truncated; however, activity was severely diminished when $\geq 1131 \mathrm{bp}$ of the $5^{\prime}$-terminal sequence was removed (Figure 2b).

\section{Search for Neural/Neuroendocrine Phenotype-Specific Transcription Factor-Binding Sites Near the Transcription Start Site of the TTF1 Gene Promoter}

We searched for transcription factors that bind to the region adjacent to the transcription start site of the TTF1 isoform 2 gene using computer software (MatInspector, Genomatix) and referring to previous reports. Figure 3 a shows a diagram of the region adjacent to the transcriptional start site of the TTF1 isoform 2 gene and potent transcription factor-binding sites. We found that LHXs and BRN2, which have been reported to be important transcription factors in developing neural cells, potentially bind to the region adjacent to the transcription start site of the TTF1 isoform 2 gene.

We next investigated the expression status of FOXA1 and 2, LHXs, and BRN2 in cultured lung cancer cells. As shown in Figure 3b, FOXA1 was expressed in all SCLC cell lines and in all adenocarcinoma and squamous cell carcinoma cell lines examined. However, in large-cell carcinoma cells, the signals were faint compared with the other cell lines. FOXA2 was expressed abundantly in most SCLC and adenocarcinoma cell lines and in one of two squamous cell carcinoma cell lines. Except for the SCLC cell lines, abundant FOXA1/FOXA2 expression did not entirely correspond with TTF1 expression. In contrast to FOXA1 and FOXA2, BRN2 expression was strictly limited to the SCLC cell lines. All SCLC cell lines examined expressed abundant BRN2. Although LHX2 and LHX6 were frequently expressed in the SCLC cell lines, some of the NSCLC cell lines also expressed LHX2, and three of eight SCLC cell lines did not express LHX6. Three SCLC cell lines modestly or weakly expressed LHX8, and two of eight NSCLC cell lines also weakly expressed LHX8. LHX1, LHX3, LHX4, LHX5, and LHX9 were not expressed in the lung cancer cell lines examined (data not shown). Weak GATA6 signals were detected in six of eight SCLC cell lines and in seven of eight NSCLC cell lines.

To examine the importance of potent FOXA1/FOXA2- and LHX/BRN2-binding sites upstream of the TTF1 gene, we next conducted TTF1 promoter mutation analyses. The luciferase activity induced by potent FOXA1/FOXA2-binding site-mutant or potent LHX/BRN2-binding site-mutant TTF1 promoters decreased $\sim 28-35 \%$ and $85-90 \%$, respectively, compared with that induced by the full-length TTF1 promoter (Figure 3c).

\section{TTF1 Gene Activation by Transfection of Candidate Regulators}

To examine whether the transcription factors mentioned above activate the TTF1 gene, we constructed FOXA1, FOXA2, LHX2, LHX6, and BRN2 retroviral vectors and transfected them into TKB5 cells. The desired gene products were sufficiently induced by $48 \mathrm{~h}$ of doxycycline treatment 


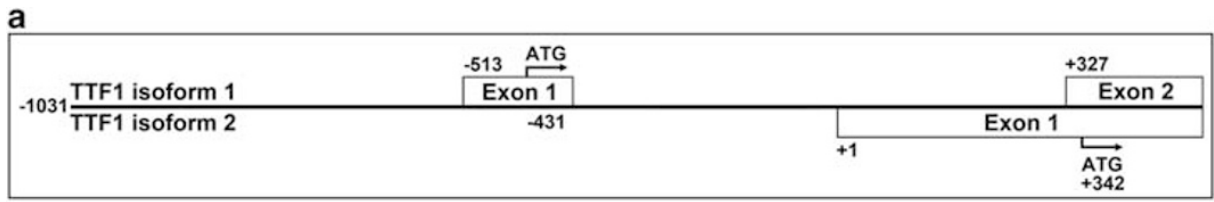

b

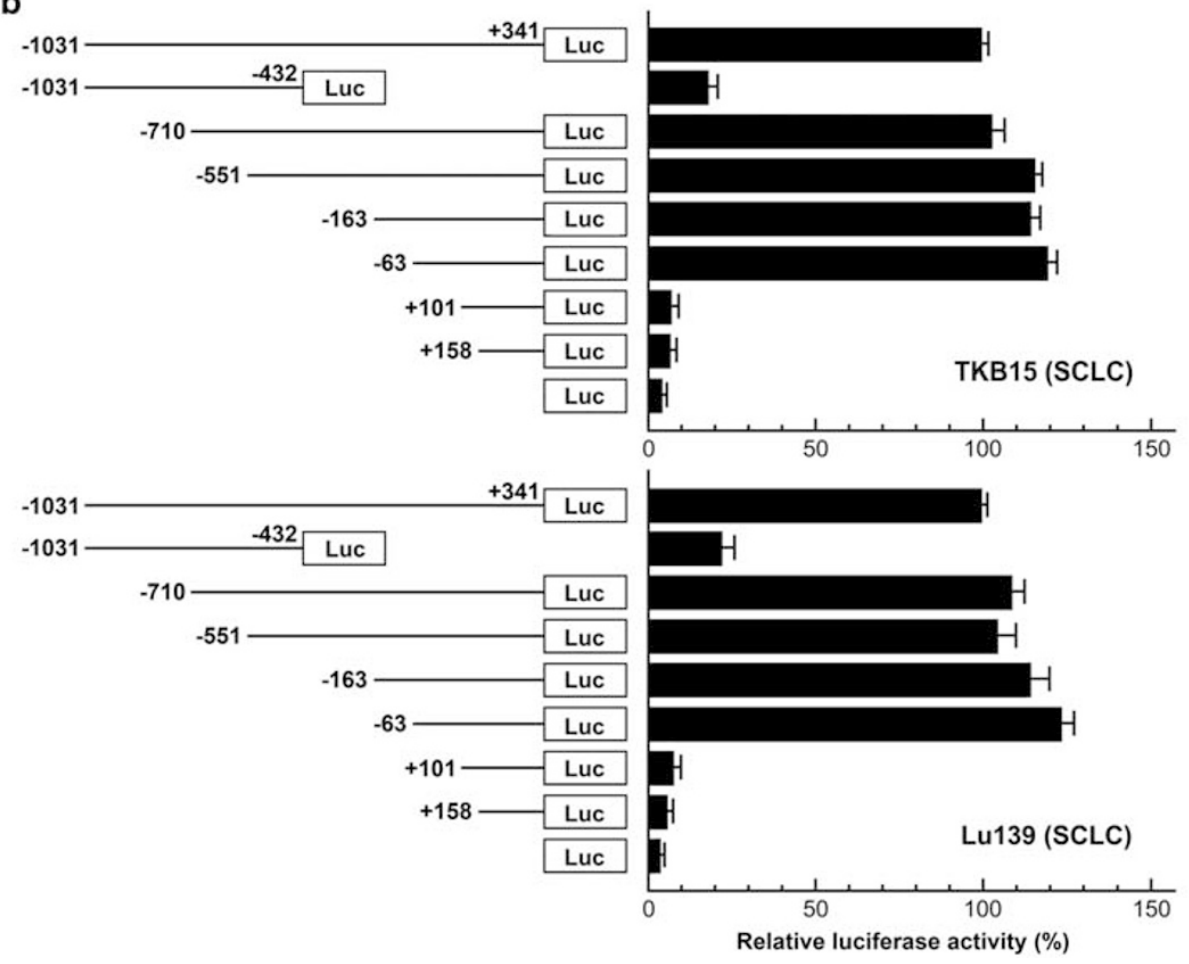

Figure 2 TTF1 promoter assay of small-cell lung cancer cell lines TKB15 and Lu139. (a) A schematic diagram of the TTF1 promoter region. (b) The promoter activity of TTF1 isoform 1 ( -1031 to -432 ) was $\sim 18-22 \%$ compared with the full-length promoter $(-1031$ to +341$)$. The luciferase activity was maintained with a 967-bp truncation of the $5^{\prime}$-terminal sequence of $\pi F 1$ gene promoter. However, the luciferase activity of 1131 bp- and 1188 bp-truncated TTF1 gene promoter sequence-inserted luciferase vector markedly decreased. Luc, luciferase cDNA.

(Figure 4a). In TKB5 cells, BRN2 induced the highest level of TTF1 expression, whereas TTF1 expression was weakly induced by FOXA1 and FOXA2 (Figure 4b). LHX2 and LHX6 expressed in TKB5 cells did not affect TTF1 expression (Figure 4b). TKB1 and A549 cells also upregulated TTF1 expression levels in association with forced BRN2 expression (Figure $4 \mathrm{~b}$ ). The function of the expressed BRN2 was confirmed through the downregulation of $\mathrm{CDH} 13$ in pBRN2transfected TKB5, TKB1, and A549 cells (Supplementary Figure 1).

To determine whether BRN2 and FOXA1/FOXA2 bind to the promoter region crucial for TTF1 gene activation, a chromatin immunoprecipitation assay using TKB15 cells was conducted. Specific PCR products were amplified from the chromatin immunoprecipitates treated with anti-BRN2, antiFOXA1, and anti-FOXA2 antibodies but not from the chromatin immunoprecipitates treated with anti-LHX2 or antiLHX6 antibodies (Figure 4c). The importance of BRN2 in TTF1 expression in SCLC cells was confirmed using siRNA experiments. BRN2 expression in TKB15 and Lu139 SCLC cells was effectively repressed by a psiBRN2 transgene (Figure 4d), and the effect of BRN2 knockdown was validated by an increase in $\mathrm{CDH} 13$ expression in psiBRN2-transfected cells compared with psiNSC-transfected cells (Supplementary Figure 1). TTF1 expression in TKB15 and Lu139 cells was decreased $\sim 23$ and $24 \%$, respectively, after BRN2-specific siRNA expression (Figures $4 \mathrm{~d}$ and e).

\section{Epigenetic Modification of the TTF1 Promoter in SCLC Cells}

To examine whether the TTF1 gene in SCLC cells is epigenetically modulated, we conducted bisulfite sequencing and compared the methylation levels of the TTF1 promoter with those of NSCLC cells. The location of the CpG islands in the TTF1 promoter and the amplified regions used for bisulfite sequencing are shown in the top panel of Figure 5 . Because the region we examined was very large, we divided it into four segments and conducted bisulfite sequencing on each segment (site A to site D). As shown in the bottom panel of Figure 5, all SCLC cell lines showed very low levels of 
a

\begin{tabular}{|c|c|c|}
\hline-61 & $+13 \sim+17+40 \sim+44$ & +101 \\
\hline & & of TTF1 isoform 2 \\
\hline
\end{tabular}

b

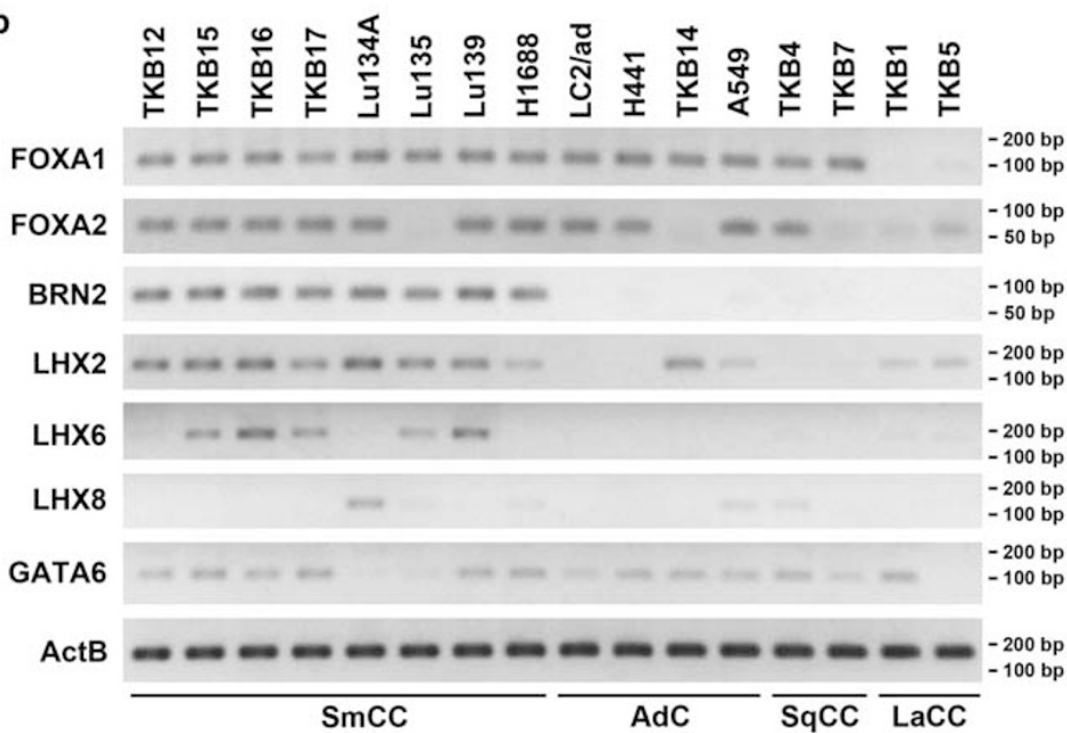

C
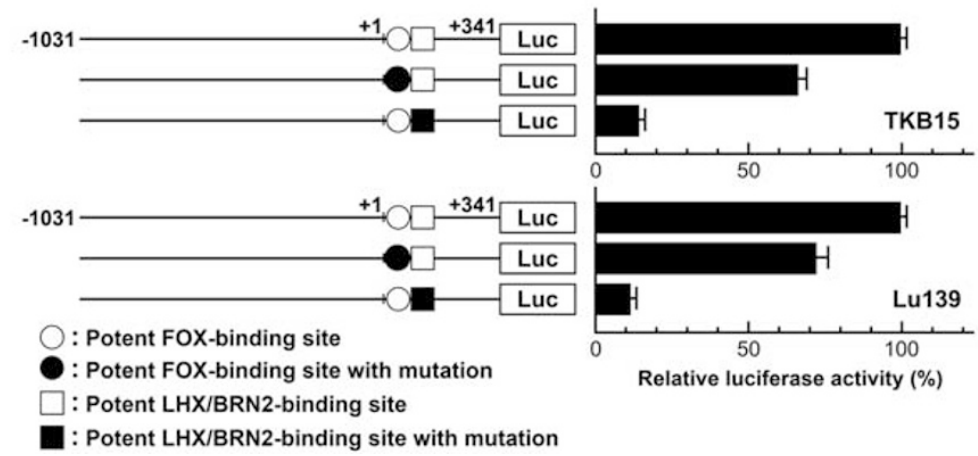

Figure 3 The search strategy used to identify potential neural/neuroendocrine phenotype-specific transcription factor binding sites located near the transcription start site of the TTF1 gene. (a) A schematic diagram of the crucial region for TTF1 gene activation. The anchor positions of potent FOX- and LHX/BRN-binding sites are indicated as an open circle $(+13$ to +17$)$ and an open box $(+40$ to +44$)$, respectively. (b) RT-PCR analyses of transcription factors expressed in cultivated lung cancer cells. Abundant FOXA1 expression was found in all lung cancer cell lines examined except for the large-cell carcinoma (LaCC) cell lines. Seven of eight small-cell carcinoma (SmCC) cell lines, three of four adenocarcinoma (AdC) cell lines, and one of two squamous cell carcinoma (SqCC) cell lines abundantly expressed FOXA2. BRN2 expression was restricted to SmCC cell lines. LHX2 expression was predominant in SmCC cell lines and was also found in some non-SmCC cell lines. LHX6 expression was observed in five of eight SmCC cell lines but was barely detectable in non-SmCC cell lines. Abundant LHX8 was expressed in only one SmCC cell line. Weak GATA6 signals were found in most $\mathrm{SmCC}$ and non-SmCC cell lines examined. $\beta$-Actin (ActB) served as an internal control. Ethidium bromide-stained gel, reverse images. (c) Mutational analyses of the TTF1 promoter. Luciferase activity in TKB15 and Lu139 cells decreased compared with cells containing the full-length TTF1 promoter after mutation of potent FOX-binding or LHX/BRN2-binding sites.

TTF1 promoter methylation, suggesting that no epigenetic modifications were present in the TTF1 gene promoter of SCLC cells. In contrast, as shown in Supplementary Figure 2, a moderate degree of $\mathrm{CpG}$ methylation was found in all sites of large-cell carcinoma cell lines (TKB1 and TKB5) and in site B of one adenocarcinoma cell line (TKB14) and one squamous cell carcinoma cell line (TKB7). Relatively heavy CpG methylation was found in site D of one squamous cell carcinoma cell line (TKB4). However, A549, which did not express detectable levels of TTF1 (Figure 2), showed scarce CpG methylation in the TTF1 promoter.

To define whether TTF1 promoter methylation and histone deacetylation were involved in TTF1 expression and to what extent, we examined TTF1 expression alterations in TTF1-deficient lung cancer cell lines (TKB14, A549, TKB4, TKB7, TKB1, and TKB5) treated with AZA or TSA. Treatment with AZA or TSA weakly increased TTF1 expression levels in all TTF1-deficient cell lines (Supplementary 


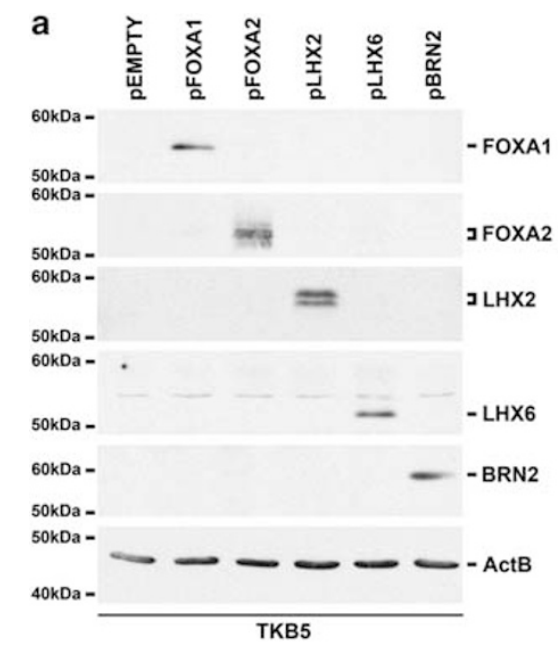

C
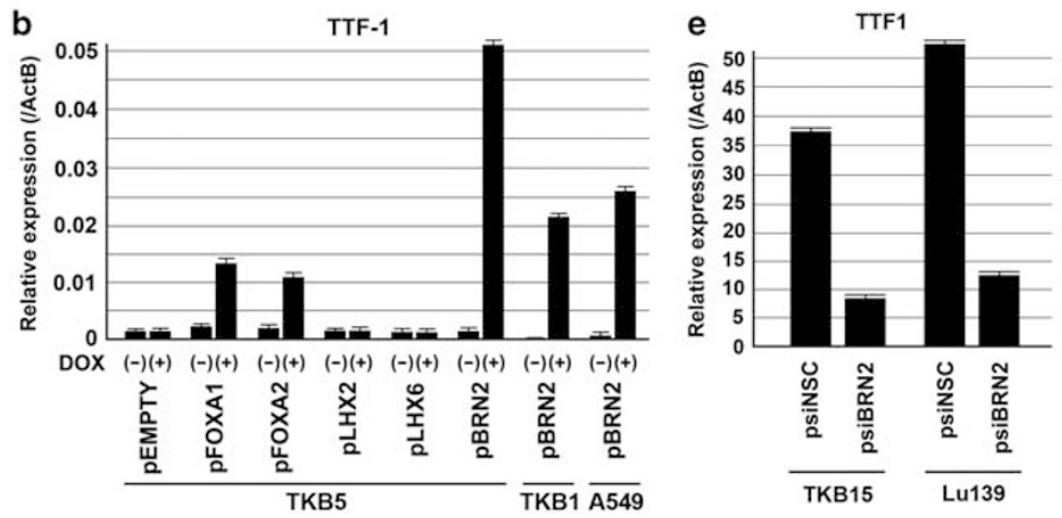

Figure 4 TTF1 gene activation by transfection of candidate genes. (a) Western blot analyses using pEMPTY-, pFOXA1-, pFOXA2-, pLHX2-, pLHX6-, or pBRN2-transfected TKB5 cells. It was confirmed that precise-sized FOXA1, FOXA2, LHX2, LHX6, and BRN2 molecules were induced by doxycycline treatment. $\beta$-Actin (ActB) was served as an internal control. (b) Changes in TTF1 mRNA expression levels in association with induction of FOXA1, FOXA2, LHX2, LHX6, or BRN2. TTF1 expression was weakly upregulated by FOXA1 and FOXA2 and strongly induced by BRN2 in TKB5 cells. TKB1 and A549 cells transfected with pBRN2 also promoted TTF1 expression through doxycycline treatment. (c) A chromatin immunoprecipitation assay using TKB15 cells. The region that included the potent FOX-binding site and the potent LHX/BRN2-binding site was amplified with PCR using a specific primer set and DNA samples that were co-immunoprecipitated with anti-FOXA1, anti-FOXA2, or anti-BRN2 antibodies. Ethidium bromide-stained gel, reverse image. (d) A BRN2 knockdown experiment. RT-PCR was conducted using mRNA extracted from BRN2-specific siRNA vector (psiBRN2)-transfected and nonspecific scrambled control vector (psiNSC)-transfected TKB15 and Lu139 cells. The BRN2 and TTF1 mRNA expression levels in psiBRN2 transfectants were considerably decreased. Ethidium bromide-stained gel, reverse image. (e) A BRN2 knockdown experiment. The results of qRT-PCR revealed that TTF1 mRNA in TKB15 and Lu139 was markedly decreased by BRN2 knockdown.

Figure 3). However, the increased TTF1 expression levels were quite low compared with BRN2-expressing TKB5, TKB1, and A549 cells (Figure 4b).

\section{Expression of TTF1, BRN2, FOXA1, FOXA2, LHX2, and LHX6 in Lung Cancer Tissues}

We conducted immunohistochemistry staining using antibodies against TTF1, BRN2, FOXA1, FOXA2, LHX2, and LHX6 and a series of primary lung tumor tissues to validate the results obtained from the in vitro experiments. The results are summarized in Table 3 , and representative immunohistochemistry pictures using consecutive SCLC tissue sections are shown in Figure 6. TTF1 expression was found in all SCLCs and adenocarcinomas examined. TTF1 revealed diffuse immunopositivity in clusters of SCLC cells as well as BRN2, FOXA1, and FOXA2 (Figure 6), and BRN2 expression was found only in SCLCs among the tumors examined. Although diffuse immunopositive staining of FOXA1 and FOXA2 was found in all adenocarcinomas, squamous cell carcinomas, and typical carcinoid tumors examined, TTF1 signals were not detected in squamous cell carcinomas and typical carcinoid tumors. LHX2 expression was observed in all SCLCs and two of two typical carcinoid tumors, and LHX6 expression was noted in four of five SCLCs and one of two typical carcinoid tumors. However, the positive cells for LHX2 or LHX6 in those tumors accounted for $<10 \%$ of tumor cells (Figure 6).

\section{DISCUSSION}

In this study, we investigated TTF1 gene expression regulation in SCLC and focused upon the associations of developmental neural cell lineage-specific transcription 

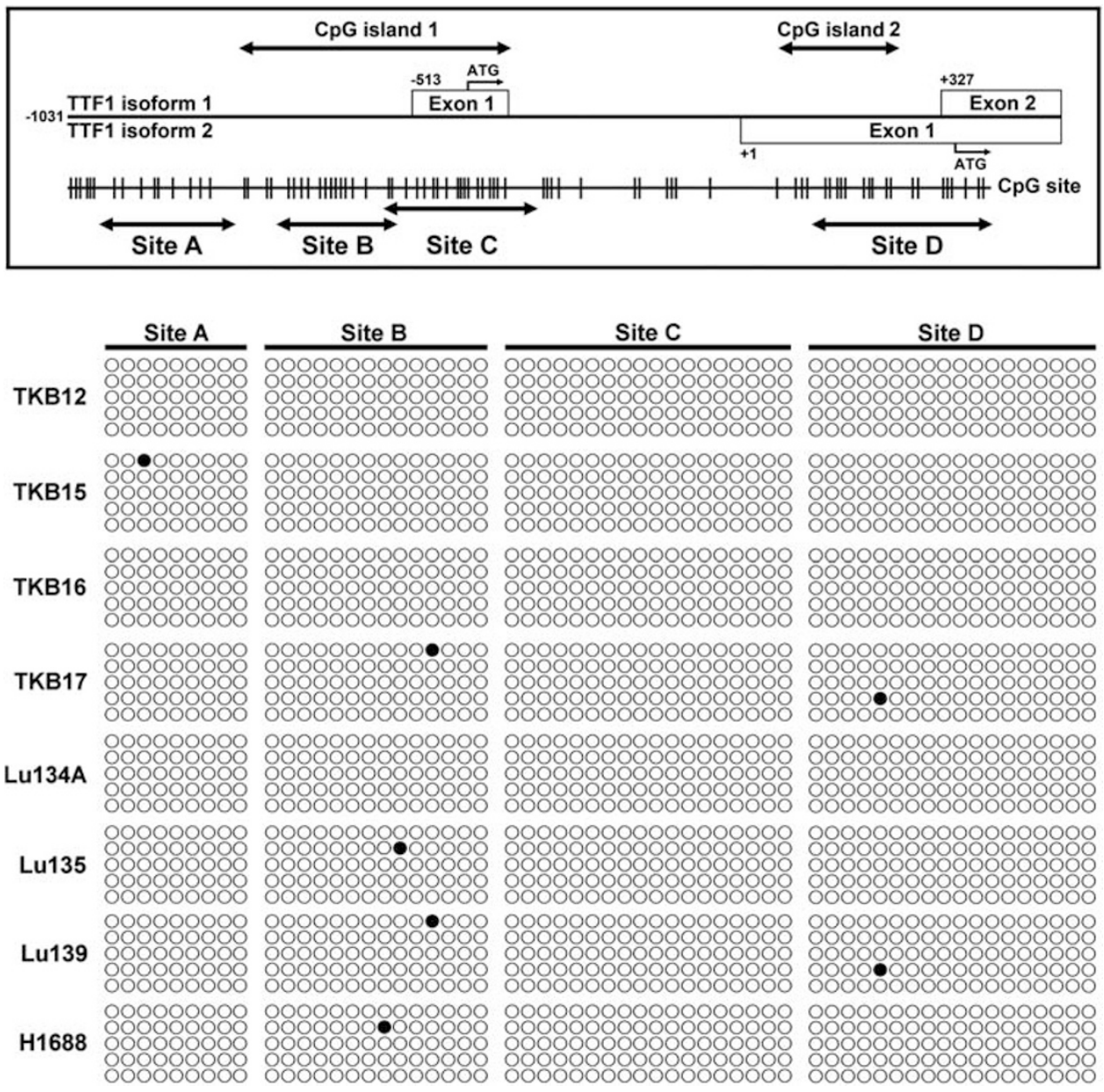

Figure 5 Epigenetic modification of the TTF1 promoter in cultivated small-cell carcinoma cells. A schematic diagram showing CpG sites, the location of CpG islands, and bisulfite sequencing sites (site A to site D) in the TTF1 promoter region (top) and the results of bisulfite sequencing (bottom). In all small-cell carcinoma cell lines examined, the cytosines in CpG sites located in the TTF1 promoter were only slightly methylated. Open circles, unmethylated cytosines; closed circles, methylated cytosines.

Table 3 Summary of immunohistochemistry in primary lung tumors

\begin{tabular}{|c|c|c|c|c|c|c|c|c|c|c|c|c|c|c|c|c|c|c|}
\hline \multirow[t]{3}{*}{ Histologic type (no. of cases) } & \multicolumn{18}{|c|}{ Immunohistochemical score } \\
\hline & \multicolumn{3}{|c|}{ TTF1 } & \multicolumn{3}{|c|}{ BRN2 } & \multicolumn{3}{|c|}{ FOXA1 } & \multicolumn{3}{|c|}{ FOXA2 } & \multicolumn{3}{|c|}{$\mathrm{LHX} 2$} & \multicolumn{3}{|c|}{ LHX6 } \\
\hline & 0 & 1 & 2 & 0 & 1 & 2 & 0 & 1 & 2 & 0 & 1 & 2 & 0 & 1 & 2 & 0 & 1 & 2 \\
\hline Small-cell carcinoma (5) & 0 & 0 & 5 & 0 & 0 & 5 & 0 & 0 & 5 & 0 & 0 & 5 & 0 & 5 & 0 & 1 & 4 & 0 \\
\hline Carcinoid tumor (2) & 2 & 0 & 0 & 2 & 0 & 0 & 0 & 0 & 2 & 0 & 0 & 2 & 0 & 2 & 0 & 1 & 1 & 0 \\
\hline Adenocarcinoma (5) & 0 & 0 & 5 & 5 & 0 & 0 & 0 & 0 & 5 & 0 & 0 & 5 & 5 & 0 & 0 & 5 & 0 & 0 \\
\hline Squamous cell carcinoma (5) & 5 & 0 & 0 & 5 & 0 & 0 & 0 & 0 & 5 & 0 & 0 & 5 & 5 & 0 & 0 & 5 & 0 & 0 \\
\hline
\end{tabular}

Score 0 , no expression in tumor cells; score 1, expression in $1-50 \%$ of tumor cells; score 2 , expression in $>50 \%$ of tumor cells.

factors and epigenetic alterations of the TTF1 promoter. We found that isoform 2 of TTF1 is mainly expressed in lung cancer cells as reported previously, ${ }^{33}$ and the TTF1 promoter assay results revealed that promoter sequences adjacent to the transcription start site of the isoform 2 gene are important for TTF1 expression in SCLC cells. Consequently, we defined that BRN2, which is a class III POU domain transcription factor and is involved in morphogenesis of diencephalon where TTF1 is specifically expressed, binds to and activates the TTF1 gene. We also revealed that the TTF1 promoter is 


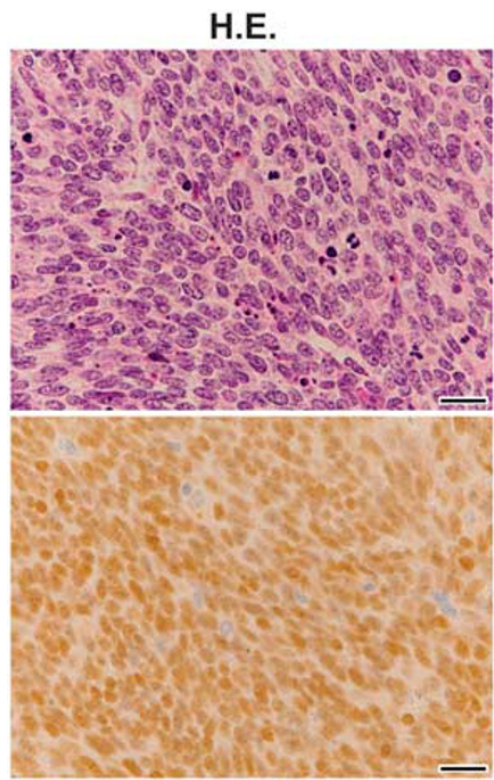

FOXA1

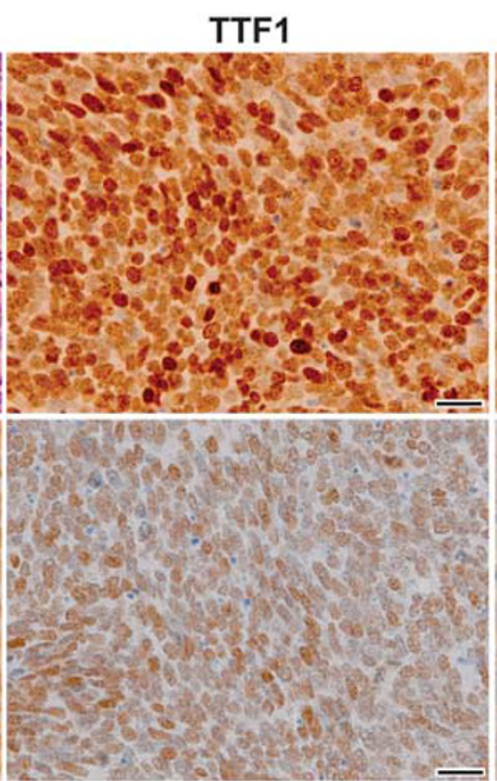

FOXA2

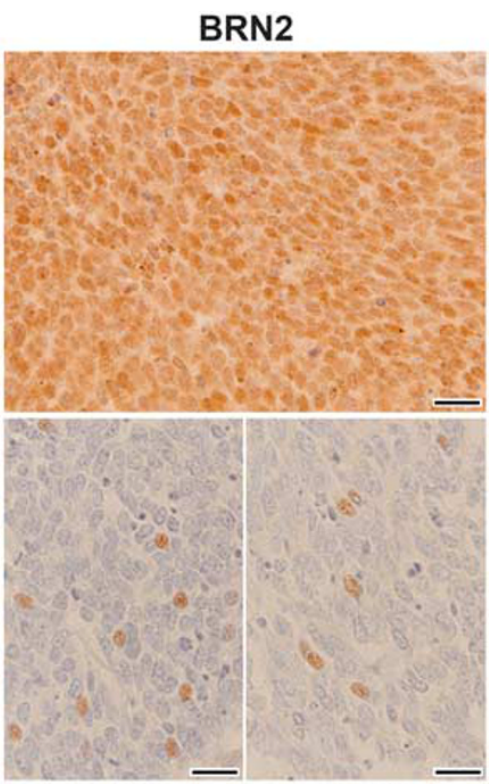

LHX2

\section{LHX6}

Figure 6 Immunohistochemistry images using consecutive tissue sections of a small-cell lung cancer. TTF1, BRN2, FOXA1, and FOXA2 were diffusely expressed in the nuclei of small-cell lung cancer cells, whereas the nuclei of a small number of small-cell lung cancer cells showed positive staining for LHX2 and LHX6. HE, hematoxylin and eosin stain; bar $=20 \mu \mathrm{m}$.

scarcely methylated in SCLC cells. Furthermore, immunohistochemical studies confirmed that BRN2 and TTF1 are coexpressed in SCLC cells.

It has been reported that FOXA1/2 contributes strongly to lung morphogenesis and regulates TTF1 expression in the peripheral airway epithelium. ${ }^{34}$ We examined the expression of FOXA1/2 using cultured lung cancer cells and primary lung tumor tissues and found that SCLC and NSCLC cells express abundant FOXA1/2. ${ }^{35}$ However, we found discrepancies in the relationship between FOXA1/2 and TTF1 expression; all SCLC and adenocarcinoma cell lines expressing TTF1 showed FOXA1/2 expression, whereas there were FOXA1/2-expressing adenocarcinoma and squamous cell carcinoma cell lines without TTF1 expression. These findings indicate the existence of further undetermined indispensable transactivators or transrepressors for TTF1 expression. Our study may help define the mechanism of TTF1 expression in NSCLC cells in more detail because there are many potent binding sites for homeoproteins, which play important roles in the determination of cell fate and cell lineage, in the crucial promoter region we identified for TTF1 isoform 2 expression.

Because the TTF1 promoter possesses CpG islands, which are sensitive to epigenetic alteration, we investigated the methylation status of the TTF1 promoter region in cultivated SCLC cells using bisulfite sequencing. We found that the TTF1 promoter region is scarcely methylated in SCLC cells, suggesting that there is no epigenetic control of the TTF1 gene in these cells. We previously investigated the mechanism of expression of a gene, the insulin-like growth factor binding protein 2 (IGFBP2) gene, which possesses CpG islands in its promoter and regulates the function of IGF via direct IGF binding. ${ }^{27,36}$ Although the IGFBP2 gene is expressed in both NSCLC and SCLC, striking differences exist in the activating mechanisms and epigenetic modification of this gene in the two cell types. The IGFBP2 gene in NSCLC cells is activated by a transactivating factor, EGR1, and IGFBP2 gene expression is epigenetically silenced in these cells by the hypermethylation of the promoter in association with cancer progression. ${ }^{36}$ In contrast, SCLC cells overexpress IGFBP2 as a result of promoter activation via binding of a neural/ neuroendocrine cell-specific basic helix-loop-helix transcription factor, NeuroD1, to the E-box, and the IGFBP2 gene promoter in SCLC is hardly methylated. ${ }^{27}$ The results of the present study and our previous studies suggest that the TTF1 gene in SCLC might, like the IGFBP2 gene, be unaffected by epigenetic modifications that maintain the faithful transmission of cell type-specific gene expression patterns.

In addition, we observed the results that there was not a perfect correlation between TTF1 expression and methylation status of the TTF1 promoter in NSCLC cells (Supplementary Figure 2) and that the expression levels of TTF1 in NSCLC cells induced by ectopic BRN2 was much higher than those recovered by treatment of AZA or TSA (Figure $4 \mathrm{~b}$ and Supplementary Figure 3). These findings suggest that extreme variation of TTF1 expression among histologic types of lung cancers is not primarily caused by epigenetic regulation but by specific transcription factors involving TTF1 expression.

Immunohistochemical analysis using a panel of primary lung tumors demonstrated that both TTF1 and BRN2 were coexpressed only in SCLCs, whereas typical carcinoid tumors, 
which are classified into low-grade malignant pulmonary neuroendocrine neoplasms, did not express these transcription factors (Table 3). It has been reported that TTF1 is not expressed in low-grade malignant pulmonary neuroendocrine neoplasms (carcinoid tumors), ${ }^{37}$ and our immunohistochemical results are consistent with this report.

Our in vitro and immunohistochemistry studies defined that, among lung tumors, SCLC specifically expresses BRN2. Taken together with previous reports, it is hypothesized that SCLC is a unique neoplasm expressing BRN2 and ASCL1, both of which are normally expressed in developing neural cells. Recently, it has been reported that a combinational transgene of BRN2, ASCL1, and MYT1L (myelin transcription factor 1-like) converts fibroblasts to functional neurons, suggesting the importance of BRN2 in transforming cells to a neural cell lineage. ${ }^{38}$ Cook et al ${ }^{39}$ reported that BRN2 acts to regulate growth and tumorigenesis in malignant melanomas, which are highly malignant neuroectodermal tumors. Therefore, BRN2 might participate in defining the cellular characteristics of SCLC cells as developing neural cells with high growth ability. Further investigations will be necessary to define the contributions of BRN2 to tumorigenesis and to SCLC progression.

In conclusion, we present here the molecular basis of TTF1 expression in SCLC. We found that a developing neural cell lineage-specific transcription factor, BRN2, was specifically expressed in SCLC among lung tumors and was involved in TTF1 expression. This finding indicates that TTF1 expression in SCLC is a lineage-specific phenomenon.

Supplementary Information accompanies the paper on the Laboratory Investigation website (http://www.laboratoryinvestigation.org)

\section{ACKNOWLEDGEMENTS}

We thank Ms Michiru Umino and Ms Ayumi Sumiishi for technical support. This study was supported by Grants-in-aid for Scientific Research from the Ministry of Education, Culture, Sports, Science, and Technology of Japan (21590376 and 24590428 to Takuya Yazawa).

\section{DISCLOSURE/CONFLICT OF INTEREST}

The authors declare no conflict of interest.

1. Lazzaro $D$, Price $M$, De Felice $M$, et al. The transcription factor TTF-1 is expressed at the onset of thyroid and lung morphogenesis and in restricted regions of the fetal brain. Development 1991;113: 1093-1104.

2. Kimura $S$, Hara $Y$, Pineau $T$, et al. The T/ebp null mouse: thyroidspecific enhancer binding protein is essential for the organogenesis of the thyroid, lung, ventral forebrain, and pituitary. Genes Dev 1996;10:60-69.

3. Minoo $P$, Su G, Drum $H$, et al. Defects in tracheoesophageal and lung morphogenesis in Nkx2.1-/- mouse embryos. Dev Biol 1999;209:60-71.

4. Civitareale D, Castelli MP, Falasca P, et al. Thyroid transcription factor 1 activates the promoter of the thyrotropin receptor gene. Mol Endocrinol 1993;7:1589-1595.

5. Bruno MD, Bohinski RJ, Huelsman KM, et al. Lung cell-specific expression of the murine surfactant protein A (SP-A) gene is mediated by interactions between the SP-A promoter and thyroid transcription factor-1. J Biol Chem 1995;270:6531-6536.

6. Zhang L, Whitsett JA, Strip BR. Regulation of Clara cell secretory protein gene transcription by thyroid transcription factor-1. Biochem Biophys Acta 1997;1350:359-367.

7. Nakamura N, Miyagi $E$, Murata $S$, et al. Expression of thyroid transcription factor- 1 in normal and neoplastic lung tissues. Mod Pathol 2002;15:1058-1067.

8. Katoh R, Kawaoi A, Miyagi E, et al. Thyroid transcription factor-1 in normal, hyperplasitc, and neoplastic follicular thyroid cells examined by immunohistochemistry and nonradioactive in situ hybridization. Mod Pathol 2000;13:570-576.

9. Ordonez NG. Thyroid transcription factor-1 is a marker of lung and thyroid carcinomas. Adv Anat Pathol 2000;7:123-127.

10. Kaufmann O, Dietel M. Thyroid transcription factor-1 is the superior immunohistochemical marker for pulmonary adenocarcinomas and large cell carcinomas compared with surfactant proteins A and B. Histopathology 2000;36:8-16.

11. Pelosi G, Fraggetta F, Pasini F, et al. Immunoreactivity for thyroid transcription factor-1 in stage I non-small cell carcinomas of the lung. Am J Surg Pathol 2001;25:363-372.

12. Yatabe $Y$, Mitsudomi T, Takahashi T. TTF-1 expression in pulmonary adenocarcinomas. Am J Surg Pathol 2002;26:767-773.

13. Maeshima AM, Omatsu M, Tsuta K, et al. Immunohistochemical expression of TTF-1 in various cytologic subtypes of primary lung adenocarcinoma, with special reference to intratumoral heterogeneity. Pathol Int 2008;58:31-37.

14. Travis WB, Nicholson S, Hirsch FR, et al. Small cell carcinoma. In: Travis WB, Brambilla E, Muller-Hermelink HK, Harris CC (eds) World Health Organization Classification of Tumours. Pathology and Genetics of Tumours of the Lung, Pleura, Thymus, and Heart, Lyon, 2004) pp31-34.

15. Folpe AL, Gown AM, Lamps LW, et al. Thyroid transcription factor-1: immunohistochemical evaluation in pulmonary neuroendocrine tumors. Mod Pathol 1999;12:5-8.

16. Ikeda K, Shaw-White JR, Wert SE, et al. Hepatocyte nuclear factor 3 activates transcription of thyroid transcription factor 1 in respiratory epithelial cells. Mol Cell Biol 1996;16:3626-3636.

17. Shaw-White JR, Bruno MD, Whitsett JA. GATA-6 activates transcription of thyroid transcription factor-1. J Biol Chem 1999;274:2658-2664.

18. Calbo J, Meuwissen R, van Montfort E, et al. Genotype-phenotype relationships in a mouse model for human small-cell lung cancer. Cold Spring Harb Symp Quant Biol 2005;70:225-232.

19. Park KS, Liang MC, Raiser DM, et al. Characterization of the cell of origin for small cell lung cancer. Cell Cycle 2011;10:2806-2815.

20. Ball DW, Azzoli CG, Baylin SB, et al. Identification of a human achaetescute homolog highly expressed in neuroendocrine tumors. Proc Natl Acad Sci USA 1993;90:5648-5562.

21. Nakai $\mathrm{S}$, Kawano $\mathrm{H}$, Yudate $\mathrm{M}$, et al. The POU domain transcription factor Brn-2 is required for the determination of specific neuronal lineages in the hypothalamus of the mouse. Genes Dev 1995;9: 3109-3121.

22. Schonemann MD, Ryan AK, McEvilly RJ, et al. Development and survival of the endocrine hypothalamus and posterior pituitary gland requires the neuronal POU domain factor Brn-2. Genes Dev 1995;9:3122-3135.

23. Schreiber E, Himmelmann A, Malipiero U, et al. Human small cell lung cancer expresses the octamer DNA-binding and nervous systemspecific transcription factor N-Oct3 (Brain-2). Cancer Res 1992;52:6121-6124.

24. Das PM, Singal R. DNA methylation and cancer. J Clin Oncol 2004;22:4632-4642.

25. Kondo T, Nakazawa T, Ma D, et al. Epigenetic silencing of TTF-1/NKX21 through DNA hypermethylation and histone $\mathrm{H} 3$ modulation in thyroid carcinomas. Lab Invest 2009;89:791-799.

26. Shimoyamada $H$, Yazawa $T$, Sato $H$, et al. Early growth response-1 induces and enhances vascular endothelial growth factor-A expression in lung cancer cells. Am J Pathol 2010;177:70-83.

27. Yazawa T, Sato $H$, Shimoyamada $H$, et al. Neuroendocrine cancer-specific up-regulating mechanism of insulin-like growth factor binding protein-2 in small cell lung cancer. Am J Pathol 2009;175:976-987. 
28. Yazawa T, Kamma H, Fujiwara $\mathrm{M}$, et al. Lack of class II transactivator causes severe deficiency of HLA-DR expression in small cell lung cancer. J Pathol 1999;187:191-199.

29. Tomita T, Nagase T, Ohga $E$, et al. Molecular mechanisms underlying human beta-defensin-2 gene expression in a human airway cell line (LC2/ad). Respirology 2002;7:305-310.

30. Bohinski RJ, Di Lauro R, Whitsett JA. The lung-specific surfactant B gene promoter is a target for thyroid transcription factor 1 and hepatocyte nuclear factor 3, indicating common factors for organ-specific gene expression along the foregut axis. Mol Cell Biol 1994;14:5671-5681.

31. Doyle LA, Yang W, Rishi AK, et al. H19 gene overexpression in atypical multi-drug resistant cells associated with expression of a 95-kilodalton membrane glycoprotein. Cancer Res 1996;58:2904-2907.

32. Ellmann L, Joshi MB, Resink TJ, et al. BRN2 is a transcriptional repressor of CDH13 (T-cadherin) in melanoma cells. Lab Invest 2012;92:1-13.

33. Li C, Cai J, Pan Q, et al. Two functionally distinct forms of NKX2.1 protein are expressed in the pulmonary epithelium. Biochem Biophys Res Commun 2000;270:462-468.
34. Maeda Y, Dave V, Whitsett JA. Transcriptional control of lung morphogenesis. Physiol Rev 2007;87:219-244.

35. Khoor A, Stahlman MT, Johnson JM, et al. Forkhead box A2 transcription factor is expressed in all types of neuroendocrine lung tumors. Hum Pathol 2004;35:560-564.

36. Sato $H$, Yazawa $T$, Suzuki $T$, et al. Growth regulation via insulin-like growth factor binding protein-4 and -2 in association with mutant K-ras in lung epithelia. Am J Pathol 2006;169: 1550-1566.

37. Sturm N, Rossi G, Lantuejoul S, et al. Expression of thyroid transcription factor- 1 in the spectrum of neuroendocrine cell lung proliferations with special interest in carcinoids. Hum Pathol 2002;33:175-182.

38. Vierbuchen T, Ostermeier A, Pang ZP, et al. Direct conversion of fibroblasts to functional neurons by defined factors. Nature 2010;463:1035-1041.

39. Cook AL, Sturm RA. POU domain transcription factors: BRN2 as a regulator of melanocytic growth and tumourigenesis. Pigment Cell Melanoma Res 2008;21:611-626. 